Townley, Stuart ; Ilchmann, Achim ; Weiß, M.G. ; Mcclements, W. ; Ruiz, A. C. ; Owen, D. H. ; Prätzel-Wolters, D.:

Existence and learning of oscillations in recurrent neural networks

Zuerst erschienen in:

IEEE Transactions on neural networks, 11 (2000), S. $205-214$ DOI: $10.1109 / 72.822523$ 


\title{
Existence and Learning of Oscillations in Recurrent Neural Networks
}

\author{
S. Townley, A. Ilchmann, M. G. Weiß, W. Mcclements, A. C. Ruiz, D. H. Owens, and D. Prätzel-Wolters
}

\begin{abstract}
In this paper we study a particular class of $n$-node recurrent neural networks (RNN's). In the 3-node case we use monotone dynamical systems theory to show, for a well-defined set of parameters, that, generically, every orbit of the $\mathrm{RNN}$ is asymptotic to a periodic orbit. Then we investigate whether RNN's of this class can adapt their internal parameters so as to "learn" and then replicate autonomously (in feedback) certain external periodic signals. Our learning algorithm is similar to identification algorithms in adaptive control theory. The main feature of the algorithm is that global exponential convergence of parameters is guaranteed. We also obtain partial convergence results in the $\boldsymbol{n}$-node case.
\end{abstract}

Index Terms-Learning systems, monotone dynamical systems, nonlinear dynamics, recurent neural networks.

\section{INTRODUCTION}

$\mathbf{R}$ ECENTLY, there has been considerable interest in recurrent neural networks (RNN's) which exhibit periodic or chaotic dynamics. RNN's which generate stable oscillations have been used to model certain biological phenomena. See, for example, [11] and [2]. RNN's which generate chaotic dynamics can be used to model oscillations in the cortex and for controlling chaotic dynamical systems; see [3], [7], and [25].

In this paper we are mainly interested in determining whether a class of RNN's can maintain a periodic orbit, and, if so, can they be forced to learn such orbits. Such periodic orbits are meant to capture the idea that certain activities or motions are learned by repetition. In the literature, there are essentially three approaches to this problem.

The first approach considers the behavior of RNN's from a computational point of view. Reference [17] has shown that a fully interconnected five-dimensional RNN can generate a stable limit cycle. This empirical approach uses a dynamic version of the steepest descent adaptation algorithm to adapt the parameters or weights of the RNN so that, after a training period, the network replicates a predetermined periodic signal. See also [6]. This approach does not analyze the mechanism by which the periodic signal is generated nor make any attempt to characterize the set of parameter values for which the RNN has pe-

Manuscript received October 27, 1998; revised July 22, 1999 and October 7 1999. This work was supported in part by the UK EPSRC under Grant GRJ8095 and an EU Human Capital and Mobility Programme, project CHRX-CT-930402. The work of W. Mcclements was supported by Ultera Electronics Ocean Systems Ltd.

S. Townley, A. Ilchmann, and W. Mcclements are with the School of Mathematical Sciences, University of Exeter, Exeter EX4 4QE, U.K.

M. G. Weiß and D. Prätzel-Wolters are with the Graduiertenkolleg Technomathematik, Universität Kaiseralsutern, 67663 Kaiserslautern, Germany.

A. C. Ruiz is with the Centre for Systems and Control Engineering, University of Exeter, Exeter EX4 4QF, U.K.

D. H. Owens is with the Department of Automatic Control and Systems Engineering, University of Sheffield, Sheffield S1 3JD, U.K.

Publisher Item Identifier S 1045-9227(00)00905-X. riodic solutions. There is no guarantee that such a set of parameters exists.

The second approach uses Hopf bifurcation techniques to prove that certain classes of RNN generate stable limit cycles. While this approach can be used to determine parameter ranges for which such limit cycles exist, by the very nature of the Hopf theorem, these results are local, both in parameter and phase space. For results in this direction see [2], [21], and the references therein.

The third approach exploits the fact that many classes of RNN's can be regarded as monotone dynamical systems. For example, [23] has studied a RNN with a cyclic structure and the work of [12] on general "cyclic" dynamical systems can be applied to classes of cyclic RNN's. These examples lead us to believe that monotone dynamical systems theory, as developed by [9], [24], and [13], provides a powerful framework for analysing the dynamics of RNN's. However, to our knowledge, developments in this direction are limited to the examples mentioned above.

In this paper we study a particular class of $n$-node RNN's. In the 3-node case we use monotone dynamical systems theory to show, for a well-defined set of parameters, that, generically, every orbit of the RNN is asymptotic to a period orbit. Then, within a usual "learning" context of neural networks, we investigate whether RNN's of this class can adapt their internal parameters so as to "learn" and then replicate autonomously certain external periodic signals. Our learning algorithm is similar to identification algorithms in adaptive control theory. The main feature of the adaptation algorithm is that global exponential convergence of parameters is guaranteed. This is in contrast to "steepest descent"-based adaptation algorithms which only find local minima of the parameter error cost functionals. We discuss in detail the extent to which these results can be extended to the $n$-node case. In particular, we prove partial convergence results for the $n$-node case. Note that while we use an identical network structure to that in [21], our results differ on two accounts. First, in Ruiz et al. convergence of a generic orbit of the RNN to a periodic orbitis only proved to $\mathcal{O}(\epsilon)$ on time scales of $\mathcal{O}(1 / \epsilon)$. Second, in Ruiz et al. the learning algorithm is based on steepest descent techniques so that only local asymptotic convergence of parameters is guaranteed.

This paper is organized as follows. In Section II we specify the class of RNN's under consideration and make precise the notions of learning and replication. To do so we introduce the so-called teaching network and learning RNN. The teaching network provides the external periodic signal which is to be learned. In Section III we prove that the orbits of the teaching network are, generically, asymptotic to a periodic orbit. In Section IV we develop the parameter adaptation algorithm by which learning is achieved. In the 3-node case our adaptation algorithm guarantees exponential convergence. We also prove partial convergence results for the $n$-node case. In Section V we comment 


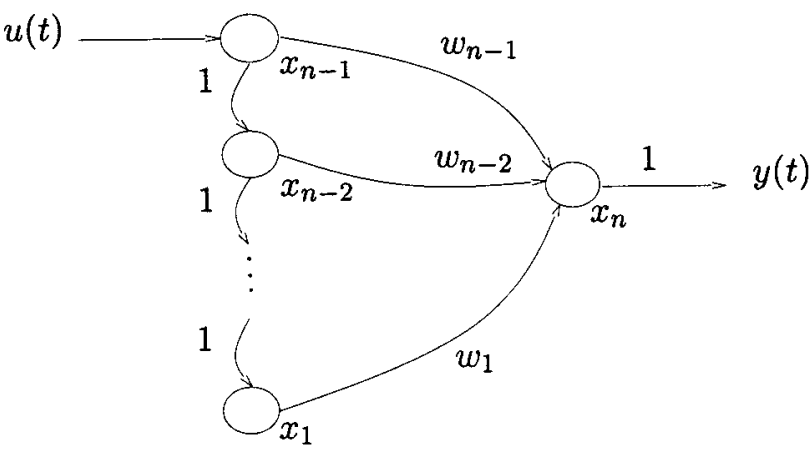

Fig. 1. Class of recurrent neural networks.

on the difficulties encountered in proving exponential convergence in the $n$-node case.

\section{StRUCTURE OF THE TEACHING NETWORK AND LEARNING RNN}

We consider the RNN shown in Fig. 1. This RNN is described formally by the system of differential equations

$$
\begin{aligned}
\dot{x}_{1}(t)= & -x_{1}(t)+\tanh x_{2}(t) \\
\vdots & \\
\dot{x}_{n-2}(t)= & -x_{n-2}(t)+\tanh x_{n-1}(t) \\
\dot{x}_{n-1}(t)= & -x_{n-1}(t)+u(t) \\
\dot{x}_{n}(t)= & -x_{n}(t)+w_{1}(t) \tanh x_{1}(t) \\
& +\cdots+w_{n-1}(t) \tanh x_{n-1}(t) \\
y(t)= & \tanh x_{n}(t)
\end{aligned}
$$

where $x(t):=\left(x_{1}(t), \cdots, x_{n}(t)\right)^{T} \in \mathbb{R}^{n}$ is the state, $x(0)=$ $x_{0} \in \mathbb{R}^{n}, w(t):=\left(w_{1}(t), \cdots, w_{n-1}(t)\right)^{T} \in \mathbb{R}^{n-1}$ is the network parameter or weight vector, $u(\cdot)$ is the input, i.e., teaching signal, and $y(\cdot)$ is the output. In (1) the nonlinear triggering function for the neurons is hyperbolic tangent. However, any triggering function, with similar properties of oddity, boundedness, monotonicity and smoothness, could have been considered. These properties are used in the proof of Lemma 3.3. In Proposition 5.1 we use a triggering function $a \mapsto \tanh \lambda a$. We are interested in whether, by adapting the weights, the RNN (1) can learn and then replicate a periodic teaching signal $u(\cdot)$. The use of a periodic teaching signal is motivated by the idea that most learning systems need repetition. To make the problem solvable we restrict the class of signals that are to be learned. In fact, we assume that the signal $u(\cdot)$ to be learned is given by

where

$$
u(t)=\tanh z_{n}(t)
$$

$$
\begin{aligned}
\dot{z}_{1}(t) & =-z_{1}(t)+\tanh z_{2}(t) \\
& \vdots \\
\dot{z}_{n-2}(t) & =-z_{n-2}(t)+\tanh z_{n-1}(t) \\
\dot{z}_{n-1}(t) & =-z_{n-1}(t)+\tanh z_{n}(t) \\
\dot{z}_{n}(t) & =-z_{n}(t)+w_{1}^{*} \tanh z_{1}(t) \\
& +\cdots+w_{n-1}^{*} \tanh z_{n-1}(t) .
\end{aligned}
$$

We refer to (2) as the teaching network. The teaching network, with state $z(t):=\left(z_{1}(t), \cdots, z_{n}(t)\right)^{T} \in \mathbb{R}^{n}$ and $z(0):=z_{0} \in$ $\mathbb{R}^{n}$, has a similar structure to (1) but the corresponding weight vector $w^{*}:=\left(w_{1}^{*}, \cdots, w_{n-1}^{*}\right)^{T}$ is fixed and the loop from $y(t)$ to $u(t)$ is closed with unity feedback. We will see in Section III that the teaching network can have periodic solutions, which we can then use as periodic teaching signals.

The RNN (1) will operate in two modes-as a learning RNN in the learning phase and as a replicating RNN in the replicating phase.

1) As a learning RNN, (1) has time-varying weights and the input $u(t)$ is equal to the output $\tanh z_{n}(t)$ of the teaching network. The time-varying weights of this learning RNN are adapted so as to enable learning of the periodic teaching signal $\tanh z_{n}(t)$ and unknown weights of the teaching network.

2) As a replicating RNN, (1) has fixed weights and operates in a unity feedback configuration. The output of this replicating RNN is meant to agree with the output of the teaching network.

The overall process of learning/replication is described as follows: The teaching network produces at its output an unknown periodic teaching signal. In the learning phase this signal is fed as input into the learning RNN. The weights of the learning RNN are then adapted. We use a weight adaptation algorithm which is similar to identification algorithms in adaptive control theory. After some finite time $T$, assumed long enough for the convergence to be adequate, we switch from the learning phase to the replication phase so that weight adaptation is terminated and the output of the teaching network is removed as input to the learning RNN to be replaced with its own output. The resulting replicating RNN, with fixed weight vector $w(T)$, then reproduces (approximately), as its output, the periodic teaching signal.

In the context of our learning/replication process, there are two crucial aspects. We must prove that the teaching network produces periodic signals as its output and we must be able to prove that the adapted weights of the learning RNN converge to the fixed weights of the teaching RNN. These issues are dealt with separately in Sections III and IV, respectively.

\section{Existence of Attractive Periodic Solutions In the TEACHING NETWORK}

In this section we focus on properties of a 3-node version of teaching network (2)

$$
\begin{aligned}
& \dot{z}_{1}(t)=-z_{1}(t)+\tanh z_{2}(t) \\
& \dot{z}_{2}(t)=-z_{2}(t)+\tanh z_{3}(t) \\
& \dot{z}_{3}(t)=-z_{3}(t)+w_{1}^{*} \tanh z_{1}(t)+w_{2}^{*} \tanh z_{2}(t) .
\end{aligned}
$$

We prove, for a range of weight values, that each trajectory of (3) which does not converge to the equilibrium $z=0$, converges to a periodic orbit. We do so by regarding the system (3) as a monotone dynamical system and by using techniques from monotone dynamical systems theory.

First we introduce the concept of a competitive dynamical system. 
Definition 3.1: Let $f: V \rightarrow \mathbb{R}^{n}$ be continuously differentiable on some open set $V \subset \mathbb{R}^{n}$. A system $\dot{x}=f(x)$ is said to be competitive on $V$ if and only if

$$
\frac{\partial f_{i}}{\partial x_{j}}(x) \leq 0, \quad \text { for all } i \neq j, \text { and for all } x \in V .
$$

Competitive systems are special cases of monotone systems. For a detailed study of monotone dynamical systems see [24] and the references therein. Note that we have defined the notion of a competitive system with respect to the positive orthant in $\mathbb{R}^{n}$ and the usual partial ordering: $x \ll y$ if and only if $x_{i} \leq y_{i}$ for all $i \neq j$, and $x \neq y$. The notion can be extended in an obvious way by considering other orthants in $\mathbb{R}^{n}$. The main result on competitive systems we need is the following proposition from [24].

Proposition 3.2: Let the system

$$
\dot{x}=f(x), \quad x(0)=x_{0} \in \mathbb{R}^{3}
$$

with $f$ continuously differentiable on an open set $V \subset \mathbb{R}^{3}$ be competitive on $V$. Suppose that $V$ contains a unique equilibrium point $x_{e}$ which is hyperbolic. Suppose further that $\mathcal{W}^{s}\left(x_{e}\right)$, the stable manifold at $x_{e}$, is one-dimensional and tangential at $x_{e}$ to a nonnegative vector $v$. If $x_{0} \notin \mathcal{W}^{s}\left(x_{e}\right)$ and the positive semi-orbit $\gamma^{+}\left(x_{0}\right):=\{x(t): t \geq 0\}$ has compact closure in $V$, then the $\omega$-limit set, $\omega\left(x_{0}\right)$, of $x_{0}$ is a nontrivial periodic orbit.

Note that this result, sometimes referred to as a Poincaré-Bendixson theorem for three-dimensional systems, does not generalize to higher dimensions, except in the special case of cyclic systems, see [12]. Furthermore, monotone systems theory does not provide us with any information concerning the uniqueness and stability of these periodic orbits. Nevertheless, Proposition 3.2 is a useful tool for establishing the existence of attractive periodic orbits in the teaching network (3).

We begin with a lemma which is proved in [21].

Lemma 3.3: Consider the system described by (3). If the fixed weights $w_{1}^{*}$ and $w_{2}^{*}$ satisfy

$$
2 w_{2}^{*}-w_{1}^{*} \geq 8, \quad 27 w_{1}^{* 2}-4 w_{2}^{* 3}>0
$$

then 1) the linearization of (3) about zero has one negative real eigenvalue and a pair of complex conjugate eigenvalues with positive real part and 2) the origin is a unique equilibrium of (3).

We use Lemma 3.3 in the following theorem which gives conditions for the guaranteed existence of periodic solutions in the teaching network (3).

Theorem 3.4: Consider the teaching network (3). Suppose that the weights satisfy (5) and, in addition, $w_{2}^{*}>0$. Then for each $z_{0} \notin \mathcal{W}^{s}(0), \omega\left(z_{0}\right)$ is a nontrivial periodic orbit.

Proof: In order to apply Proposition 3.2 we need some preliminary results.

1) We show for all $z_{0} \in \mathbb{R}^{3}$, that the positive semiorbit $\gamma^{+}\left(z_{0}\right)$ has compact closure. Indeed, since $a \mapsto \tanh a$ is bounded, we can view (3) as an exponentially stable linear system driven with a bounded input. Therefore every positive semiorbit is bounded and so has compact closure in $\mathbb{R}^{3}$. In fact the set $\left\{z \in \mathbb{R}^{3}\left|\|z\| \leq 4\left(1+\left\|w_{1}^{*}\right\|\right)+\| w_{2}^{*}\right| \mid\right\}$ attracts all solutions.

2) It follows from (5) and Lemma 3.3, parts 1) and 2), that $z_{e}=0$ is a unique hyperbolic equilibrium of (3) with a one-dimensional stable manifold $\mathcal{W}^{s}(0)$.

3) In order to apply Proposition 3.2, we transform (3) into a competitive system on $\mathbb{R}^{3}$. We do so by using a change of coordinates $\xi_{1}=-z_{1}, \xi_{2}=z_{2}, \xi_{3}=-z_{3}$ which, when applied to (3), gives

$$
\begin{aligned}
& \dot{\xi}_{1}(t)=-\xi_{1}(t)-\tanh \xi_{2}(t) \\
& \dot{\xi}_{2}(t)=-\xi_{2}(t)-\tanh \xi_{3}(t) \\
& \dot{\xi}_{3}(t)=-\xi_{3}(t)+w_{1}^{*} \tanh \xi_{1}(t)-w_{2}^{*} \tanh \xi_{2}(t) .
\end{aligned}
$$

Since the weights satisfy (5), so that in particular $w_{1}^{*}<$ 0 , and by assumption $w_{2}^{*}>0$, it follows that the right-hand side of (6) satisfies (4). Hence the system (6) is competitive.

4) Let $P$ be the Jacobian matrix of the right-hand side of (6) evaluated at zero. Clearly $-P$ is nonnegative and $(-P)^{2}$ is a positive matrix (i.e., $(-P)$ is a primitive matrix). It follows (see, for example, [10, Sec. 8.5 ]) that $-P$ has a positive eigenvector corresponding to the (unique by Lemma 3.3) negative real eigenvalue of $P$. Hence the stable manifold for the zero equilibrium of (6) is tangential at zero to a positive vector.

The proof is now complete since we can use 1)-4) to apply Proposition 3.2 to (6).

\section{Remark 3.5:}

1) The conclusions of Theorem 3.4 will hold if the triggering function $a \mapsto \tanh a$ is replaced by any other function $\sigma$ with similar properties of oddity, boundedness, monotonicity, and smoothness provided that the inequalities in (5) are scaled appropriately to account for $\sigma^{\prime}(0) \neq 1$.

2) Note that in the case $w_{1}^{*}$ and $w_{2}^{*}$ satisfying (5) but with $w_{2}^{*}<0$, we have not been able to find a change of coordinates which converts (3) into a competitive system in the sense of Definition 3.1. This has prevented us from extending Theorem 3.4 to the case $w_{2}^{*}<0$. However in [21] we have shown, for such weight parameters, a weaker convergence to a periodic function to $\mathcal{O}(\epsilon)$ on time scales of $\mathcal{O}(1 / \epsilon)$.

3) For weights satisfying the conditions of Theorem 3.4, typically the resulting teaching signal is sinusoidal in nature. This "linear-like" behavior is somewhat surprising because the teaching network is strongly nonlinear and we allow parameters far from the Hopf bifurcation curves in weight space.

Note that for any weight values satisfying (5), not only does every simulation we have tried produce solutions converging to a periodic function, but that for each pair of weights this periodic function is unique, i.e., simulations suggest that for each pair of weights satisfying (5), (3) has a limit cycle which attracts all solutions except those starting in $\mathcal{W}^{s}(0)$. It is easy to see that the solution $z(\cdot)$ of the teaching network (2) is constant if and only 
if one of its components $z_{i}(\cdot)$ is. We conclude this section with a lemma concerning the linear independency of the functions $\tanh z_{1}(\cdot)$ and $\tanh z_{2}(\cdot)$ in the case of the 3-node teaching network. Let

$$
B(t):=\left(\tanh z_{1}(t), \tanh z_{2}(t)\right)^{T} .
$$

Lemma 3.6: Assume that $z(\cdot)$ is a nontrivial periodic solution of (3) with period $\tau$. Then the functions tanh $z_{1}(\cdot)$ and $\tanh z_{2}(\cdot)$ are linearly independent or equivalently

$$
\int_{0}^{\tau} B(t) B(t)^{T} d t>0
$$

Proof: Suppose that $\tanh z_{1}(\cdot)$ and $\tanh z_{2}(\cdot)$ are dependent. Since neither of tanh $z_{1}(\cdot)$ and $\tanh z_{2}(\cdot)$ are constant, we can find $a \neq 0$ so that $\tanh z_{2}(\cdot) \equiv a \tanh z_{1}(\cdot)$. Substituting for $z_{2}$ in the first equation in (3) gives

$$
\dot{z}_{1}(t)=-z_{1}(t)+a \tanh z_{1}(t) .
$$

It then follows that $z_{1}(\cdot)$ is constant since the only periodic solution of a first-order equation is a constant solution. This implies that $z(\cdot)$ is constant which is a contradiction. Therefore $\tanh z_{1}(\cdot)$ and $\tanh z_{2}(\cdot)$ are linearly independent. Now (8) holds if and only if $\int_{0}^{\tau}\left(v^{T} B(t)\right)^{2} d t>0$ for all nonzero $v \in \mathbb{R}^{2}$, i.e., $v^{T} B(\cdot) \not \equiv 0$, for all nonzero $v \in \mathbb{R}^{2}$, i.e., if and only if $\tanh z_{1}(\cdot)$ and $\tanh z_{2}(\cdot)$ are linearly independent.

\section{Remark 3.7:}

1) The condition given by $(8)$ states that $B(\cdot)$ is persistently exciting; see [14].

2) We have been unable to obtain necessary and sufficient conditions for persistency of excitation of $\left(\tanh z_{1}(\cdot), \cdots, \tanh z_{n-1}(\cdot)\right)^{T}$, in the case of an $n$-node teaching network. In Proposition 5.1 we show, for a teaching network with triggering function $a \mapsto \tanh \lambda a$ in place of $a \mapsto \tanh a$, that persistency of excitation can fail even though the teaching network has a limit cycle. However, all our simulations suggest that persistency of excitation holds generically among those weight vectors $w^{*}$ yielding periodic solutions.

\section{LEARNING THE OUTPUT FROM THE TEACHING NETWORK}

In this section we construct weight adaptation algorithms which enable the learning RNN to learn the output of the teaching network. More accurately, in the case $n=3$ the learning algorithms guarantee that the state $x(t)$ and weight vector $w(t)$ of the learning RNN converge exponentially to the state $z(t)$ and fixed weight vector $w^{*}$ of the teaching network. The weight adaptation algorithms are similar to identification algorithms in adaptive control. The convergence proofs use the persistency of excitation property (8). We also obtain partial convergence results for the $n$-node case.

Theorem 4.1: Consider the 3-node teaching network (3) and the corresponding 3-node Learning RNN

$$
\begin{aligned}
& \dot{x}_{1}(t)=-x_{1}(t)+\tanh x_{2}(t) \\
& \dot{x}_{2}(t)=-x_{2}(t)+u(t) \\
& \dot{x}_{3}(t)=-x_{3}(t)+w_{1}(t) \tanh x_{1}(t)+w_{2}(t) \tanh x_{2}(t)
\end{aligned}
$$

where

$$
u(t)=\tanh z_{3}(t)
$$

Define the weight adaptation algorithm by

$$
\begin{aligned}
& \dot{w}_{1}(t)=-\left(x_{3}(t)-z_{3}(t)\right) \tanh x_{1}(t) \\
& \dot{w}_{2}(t)=-\left(x_{3}(t)-z_{3}(t)\right) \tanh x_{2}(t) .
\end{aligned}
$$

Then for arbitrary initial conditions $z(0), x(0) \in \mathbb{R}^{3}$ and $w(0) \in \mathbb{R}^{2}$, the closed-loop system (3), (9)-(11) has a unique solution defined on $[0, \infty)$. Furthermore, if $z(\cdot)$ is a nontrivial periodic solution of (3), then there exist $M, \lambda>0$ independent of $x(0) \in \mathbb{R}^{3}$ and $w(0) \in \mathbb{R}^{2}$, so that

$$
\left\|\left(\begin{array}{c}
x(t)-z(t) \\
w(t)-w^{*}
\end{array}\right)\right\| \leq M e^{-\lambda t} \underset{\text { for all } t \geq 0 .}{\left\|\left(\begin{array}{c}
x(0)-z(0) \\
w(0)-w^{*}
\end{array}\right)\right\|,}
$$

Proof: Existence and uniqueness of solutions on $[0, \infty)$ is guaranteed because the right-hand side of the closed-loop system (3), (9)-(11) is continuous and affine linearly bounded.

Let $e(t)=x(t)-z(t)$. Then

$$
\begin{aligned}
\dot{e}_{1}(t)= & -e_{1}(t)+\beta_{2}(t), \\
\dot{e}_{2}(t)= & -e_{2}(t), \\
\dot{e}_{3}(t)= & -e_{3}(t)+\tilde{w}_{1}(t) \tanh x_{1}(t)+\tilde{w}_{2}(t) \tanh x_{2}(t) \\
& +w_{1}^{*} \beta_{1}(t)+w_{2}^{*} \beta_{2}(t)
\end{aligned}
$$

where, for $i=1$ or $2, \beta_{i}(t):=\tanh x_{i}(t)-\tanh z_{i}(t)$ and $\tilde{w}_{i}(t):=w_{i}(t)-w_{i}^{*}$. Clearly, from (13)

$$
e_{2}(t)=e^{-t} e_{2}(0), \quad \text { for all } t \geq 0 .
$$

Since $a \mapsto \tanh a$ has a global Lipschitz constant equal to one we have that

$$
\left|\beta_{i}(t)\right| \leq\left|e_{i}(t)\right|, \quad \text { for all } t \geq 0 \text { and } i=1,2 .
$$

It follows, using variation of contants in the first equation in (13), and then taking estimates, that

$$
\begin{aligned}
\left|e_{1}(t)\right| & \leq e^{-t}\left|e_{1}(0)\right|+\int_{0}^{t} e^{-(t-s)}\left|\beta_{2}(s)\right| d s \\
& \leq e^{-t}\left|e_{1}(0)\right|+\int_{0}^{t} e^{-(t-s)}\left|e_{2}(s)\right| d s \\
& \leq e^{-t}\left|e_{1}(0)\right|+e^{-t} t\left|e_{2}(0)\right| \\
& \leq e^{-t}\left|e_{1}(0)\right|+2 e^{-t / 2} e^{-1}\left|e_{2}(0)\right| .
\end{aligned}
$$

Hence there exists $M_{1}, \lambda_{1}>0$ such that

$$
\left|e_{i}(t)\right| \leq M_{1} e^{-\lambda_{1} t}\|e(0)\| \quad \text { for all } t \geq 0 \text { and } i=1,2 .
$$

It remains to show that $\eta(t):=\left(e_{3}(t), \tilde{w}_{1}(t), \tilde{w}_{2}(t)\right)^{T}$ decays to zero exponentially. First we show that the weights are bounded. Indeed, differentiating

$$
V(t)=\frac{1}{2}\|\eta(t)\|^{2}
$$

along solutions and using (15) and (16), we obtain for all $t \geq 0$ that

$$
\begin{aligned}
\dot{V}(t) & =-e_{3}^{2}(t)+e_{3}(t)\left(w_{1}^{*} \beta_{1}(t)+w_{2}^{*} \beta_{2}(t)\right) \\
& \leq-\frac{1}{2} e_{3}^{2}(t)+\frac{1}{2}\left(\left|w_{1}^{*}\right|+\left|w_{2}^{*}\right|\right)^{2} M_{1}^{2} e^{-2 \lambda_{1} t} .
\end{aligned}
$$


Integrating (17) from zero to $t$ gives

$$
\begin{aligned}
V(t)= & \frac{1}{2}\|\eta(t)\|^{2} \leq V(0)-\frac{1}{2} \int_{0}^{t} e_{3}^{2}(s) d s \\
& +\frac{1}{2} \int_{0}^{\infty}\left(\left|w_{1}^{*}\right|+\left|w_{2}^{*}\right|\right)^{2} M_{1}^{2} e^{-2 \lambda_{1} s} d s<\infty
\end{aligned}
$$

It follows from (18) that $\eta(\cdot)$ and, in particular, $w(\cdot)$ is bounded. Next we look at the differential equation which describes the evolution of $\eta(\cdot)$. This can be written in the form

$$
\dot{\eta}(t)=A(t) \eta(t)+P(t) \eta(t)+D(t)
$$

where

$$
\begin{aligned}
A(t) & :=\left(\begin{array}{cc}
-1 & B(t)^{T} \\
-B(t) & 0
\end{array}\right) \\
P(t) & :=\left(\begin{array}{ccc}
0 & 0 & 0 \\
-\beta_{1}(t) & 0 & 0 \\
-\beta_{2}(t) & 0 & 0
\end{array}\right) .
\end{aligned}
$$

$D(t):=\left(\left[w_{1}(t) \beta_{1}(t)+w_{2}(t) \beta_{2}(t)\right], 0,0\right)^{T}$ and $B(\cdot)$ is given by (7). Notice that $P(t)$ and $D(t)$, which we consider as perturbation terms in (19) decay to zero exponentially. In analysing (19) we first consider the "unperturbed," homogeneous system

$$
\dot{\xi}(t)=A(t) \xi(t), \quad \xi(0):=\xi_{0}
$$

with $\xi(t) \in \mathbb{R}^{3}$. Since $B(\cdot)$ satisfies the persistency of excitation condition (8) it follows from Corollary 2.3 in [15], that the system given by (20) is uniformly exponentially stable. Now, using the fact that $\lim _{t \rightarrow \infty}\|P(t)\|=0$, it follows from standard perturbation results, (see, for example, [19, p. 134]), that for the transition matrix $\Psi(\cdot, \cdot)$ of

$$
\dot{\nu}(t)=(A(t)+P(t)) \nu(t)
$$

there exists $M_{2}, \lambda_{2}>0$ so that

$$
\|\Psi(t, s)\| \leq M_{2} e^{-\lambda_{2}(t-s)}
$$

for all $t \geq s$, and $s \geq 0$. Using variation of constants in (19) and estimating, using (21), gives, for all $t \geq 0$

$$
\|\eta(t)\| \leq M_{2} e^{-\lambda_{2} t} \eta(0)+\int_{0}^{t} M_{2} e^{-\lambda_{2}(t-s)}\|D(s)\| d s .
$$

Now using the boundedness of $w(\cdot)$ and the exponential decay to zero of $\beta_{1}(\cdot)$ and $\beta_{2}(\cdot)$ given by $(15)$ and (16) we have that $\|D(t)\|$ converges to zero exponentially as $t$ tends to $\infty$. It then follows that there exists $M, \lambda>0$ so that (12) holds.

Remark 4.2: Note that the learning algorithm (11) is realizable since $z_{3}(\cdot)$ can be obtained from the teaching signal $\tanh z_{3}(\cdot)$ using the invertibility of $a \mapsto \tanh a$. Fig. 2 shows the results from a simulation of the learning algorithm where the weights of the teaching signal are $w^{*}=(-20,10)$, $z(0)=(0.4736,0.8745,1.8497), x(0)=(0,0,0)$ and $w(0)=(0,0)$. The simulation shows clearly that the output of the teaching network is periodic.

The weight adaptation (11) is chosen to make the right-hand side of (17) seminegative definite, except for a term which decays to zero exponentially. Note that the algorithm (11) guarantees not only local, but global convergence. A similar construction can be used in the $n$-node case to obtain the following partial extension of Theorem 4.1.
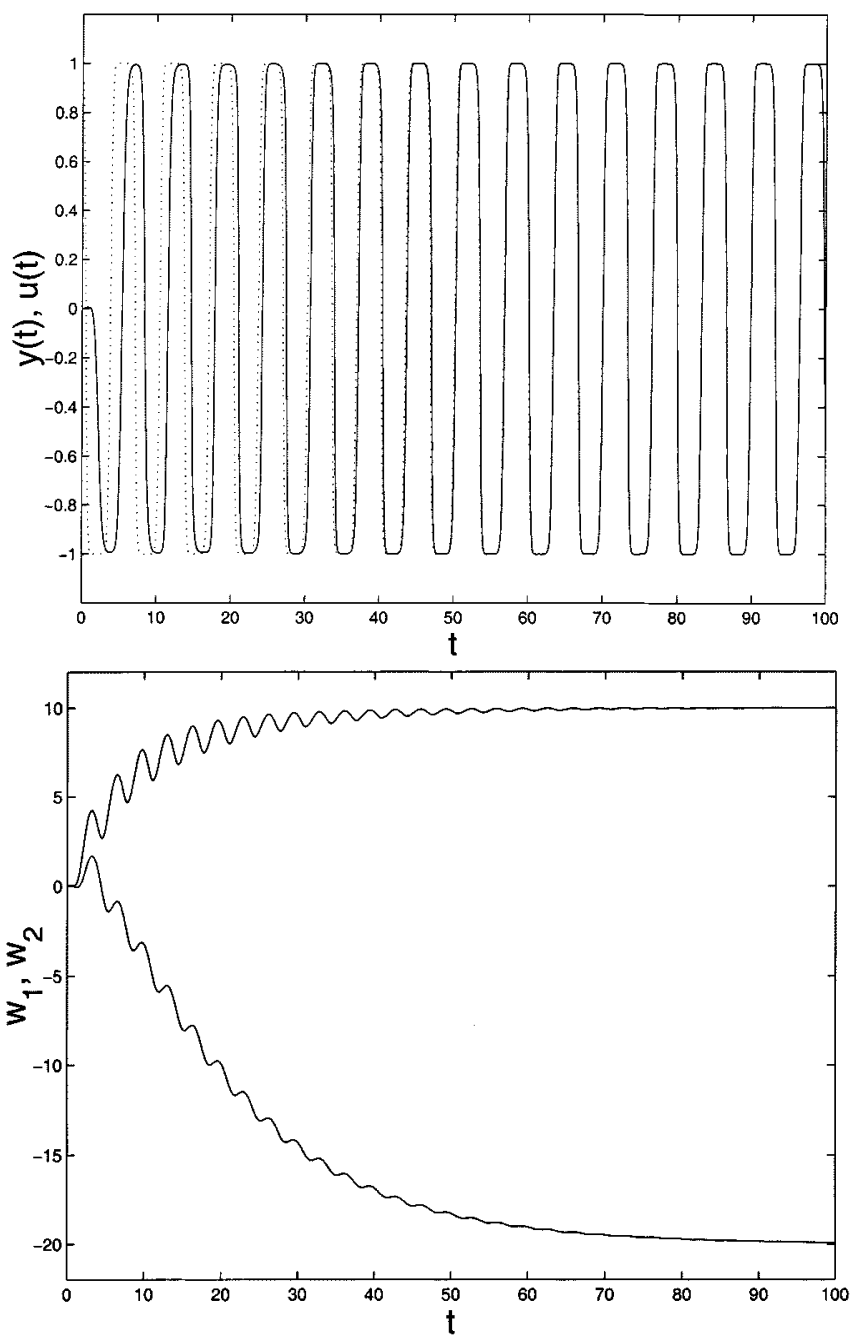

Fig. 2. (Top) reference signal $u(t)$ (dotted), learning signal $y(t)$ (continuous); (Bottom): weight dynamics $w_{1}(t)$ and $w-2(t)$.

Theorem 4.3: Let $x(t)$ and $z(t)$ be given by (1) and (2), respectively. Define the weight adaptation algorithm by

$\dot{w}_{i}(t)=-\left[x_{n}(t)-z_{n}(t)\right] \tanh x_{i}(t), \quad$ for $i=1, \cdots, n-1$.

Then

1) there exist $M, \lambda>0$ independent of $x(0), z(0)$, and $w(0)$ so that

$$
\begin{array}{r}
\left|x_{i}(t)-z_{i}(t)\right| \leq M e^{-\lambda t}||(x(0)-z(0)) \| \\
\quad \text { for all } t \geq 0 \text { and } i=1, \cdots, n-1 ;
\end{array}
$$

2) the weights $w_{i}(\cdot)$ are bounded;

3) $\lim _{t \rightarrow \infty}\left|x_{n}(t)-z_{n}(t)\right|=0$;

4) for any $t_{1}>0$ we have

$\lim _{t \rightarrow \infty} \tilde{w}(t)^{T}\left(\int_{t}^{t+t_{1}} B(s) B(s)^{T} d s\right) \tilde{w}(t)=0$

where $B(t):=\left(\tanh z_{1}(t), \cdots, \tanh z_{n-1}(t)\right)^{T}$.

Proof: As in the proof of Theorem 4.1, introduce

$$
\begin{aligned}
e(t) & :=x(t)-z(t) \\
\beta_{i}(t) & :=\tanh x_{i}(t)-\tanh z_{i}(t) \\
\tilde{w}(t) & :=w(t)-w^{*} .
\end{aligned}
$$


Then

$$
\dot{e}_{n}(t)=-e_{n}(t)+\sum_{i=1}^{n-1}\left(\tilde{w}_{i}(t) \tanh x_{i}(t)+w_{i}^{*} \beta_{i}(t)\right) \text {. }
$$

Part 1) follows analogously to the corresponding result in Theorem 4.1. To prove parts 2) and 3) let

$$
V(t)=\frac{1}{2}\|\eta(t)\|^{2}
$$

where $\eta(t):=\left(e_{n}(t), \tilde{w}_{1}(t), \cdots, \tilde{w}_{n-1}(t)\right)^{T}$. Then, as in the 3-node case

$$
\begin{aligned}
\dot{V}(t) & =-e_{n}(t)^{2}+e_{n}(t)\left[w_{1}^{*} \beta_{1}(t)+\cdots+w_{n-1}^{*} \beta_{n-1}(t)\right] \\
& \leq-\frac{1}{2} e_{n}(t)^{2}+\frac{1}{2}\left(\left|w_{1}^{*}\right|+\cdots+\left|w_{n-1}^{*}\right|\right)^{2} M^{2} e^{-2 \lambda t}
\end{aligned}
$$

and

$$
\begin{aligned}
V(t) \leq & V(0)-\frac{1}{2} \int_{0}^{t} e_{n}(s)^{2} d s \\
& +\frac{1}{2} \int_{0}^{\infty} M^{2}\left(\left|w_{1}^{*}\right|+\cdots+\left|w_{n-1}^{*}\right|\right)^{2} e^{-2 \lambda t} d t<\infty .
\end{aligned}
$$

It follows that $e_{n}(\cdot)$ and $\tilde{w}(\cdot)$ are bounded, and $e_{n}(\cdot) \in$ $L^{2}(0, \infty)$. Then, from (25), it follows that $\dot{e}_{n}(\cdot)$ is bounded. Hence, using Barbălat's Lemma (see Corollary 2.9 in [15]), $\lim _{t \rightarrow \infty} e_{n}(t)=0$. This proves 2) and 3). All that remains is to prove the partial convergence 4). To do this we borrow techniques from partial convergence proofs in adaptive control (see [22, Th. 2.7.4]. Now for each $i=1, \cdots, n-1$

$$
\lim _{t \rightarrow \infty} \dot{\widetilde{w}}_{i}(t)=-\lim _{t \rightarrow \infty}\left(e_{n}(t) \tanh x_{i}(t)\right)=0 .
$$

Let $t_{1}>0$ be arbitrary. We claim that

$$
\lim _{t \rightarrow \infty} \int_{t}^{t+t_{1}}\left|\tilde{w}(s)^{T} B(s)\right|^{2} d s=0 .
$$

Indeed, using

$$
\dot{e}_{n}(t)=-e_{n}(t)+\tilde{w}(t)^{T} B(t)+\sum_{i=1}^{n-1} w_{i}(t) \beta_{i}(t)
$$

and the facts that $e_{n}(\cdot) \in L^{2}(0, \infty), w(\cdot) \in L^{\infty}(0, \infty)$, and from 1) and the global Lipschitz continuity of $a \mapsto \tanh a$, that $\left|\beta_{i}(t)\right| \leq M e^{-\lambda t}$ for each $i=1, \cdots, n-1,(27)$ holds if

$$
\lim _{t \rightarrow \infty} \int_{t}^{t+t_{1}} \dot{e}_{n}(s)^{2} d s=0 .
$$

Now, from (25), $\dot{e}_{n}(\cdot) \in L^{\infty}(0, \infty)$ and

$$
\begin{aligned}
\ddot{e}_{n}(t)= & -\dot{e}_{n}(t)+\sum_{i=1}^{n-1}\left[\dot{\tilde{w}}_{i}(t) \tanh x_{i}(t)\right. \\
& +w_{i}(t)\left(1-\left(\tanh x_{i}(t)\right)^{2}\right) \dot{x}_{i}(t) \\
& \left.+w_{i}^{*}\left(1-\left(\tanh z_{i}(t)^{2}\right)\right) \dot{z}_{i}(t)\right]
\end{aligned}
$$

yields $\ddot{e}_{n}(\cdot) \in L^{\infty}(0, \infty)$. Then, using part 3$)$, we have

$$
\begin{aligned}
\lim _{t \rightarrow \infty} & \int_{t}^{t+t_{1}} \dot{e}_{n}(s)^{2} d s \\
=\lim _{t \rightarrow \infty} & \left(e_{n}\left(t+t_{1}\right) \dot{e}_{n}\left(t+t_{1}\right)-e_{n}(t) \dot{e}_{n}(t)\right. \\
& \left.-\int_{t}^{t+t_{1}} e_{n}(s) \ddot{e}_{n}(s) d s\right)=0
\end{aligned}
$$

and therefore (27) holds as claimed. Let $M_{1} \geq \max \left(\|B(\cdot)\|_{\infty}\right.$, $\left.\|\tilde{w}(\cdot)\|_{\infty}\right)$. Then, following the techniques in [22, proof of Th. 2.7.4], we have

$$
\begin{aligned}
\tilde{w}(t)^{T} & \left(\int_{t}^{t+t_{1}} B(s) B(s)^{T} d s\right) \tilde{w}(t) \\
= & \int_{t}^{t+t_{1}} \tilde{w}(s)^{T} B(s) B(s)^{T} \tilde{w}(s) d s \\
& +\int_{t}^{t+t_{1}}(\tilde{w}(t)-\tilde{w}(s))^{T} B(s) B(s)^{T}(\tilde{w}(t)+\tilde{w}(s)) d s \\
\leq & \int_{t}^{t+t_{1}} \tilde{w}(s)^{T} B(s) B(s)^{T} \tilde{w}(s) d s \\
& +2 M_{1}^{3} \sup _{s \in\left[t, t+t_{1}\right]}\|\dot{\tilde{w}}(s)\| \int_{t}^{t+t_{1}}|t-s| d s \\
= & \left.\int_{t}^{t+t_{1}} \operatorname{lip}^{T} \tilde{w}(s)^{T} B(s)\right|^{2} d s \\
& +M_{1}^{3} t_{1}^{2} \sup _{s \in\left[t, t+t_{1}\right]}\|\dot{\tilde{w}}(s)\| .
\end{aligned}
$$

Taking limits in (28) as $t$ tends to $\infty$, using (26) and (27), gives 4).

Corollary 4.4: Under the assumptions of Theorem 4.3:

1) If $z(\cdot)$ is periodic and $B(\cdot)$ is persistently exciting, then $e(\cdot)$ and $\tilde{w}(\cdot)$ converge to zero exponentially;

2) If $z(\cdot)$ is periodic with period $\tau$, then

$$
\lim _{t \rightarrow \infty} \tilde{w}(t)^{T}\left(\int_{0}^{\tau} B(s) B(s)^{T} d s\right) \tilde{w}(t)=0
$$

i.e., the weight vector converges $\operatorname{ker}\left(\int_{0}^{\tau} B(s) B(s)^{T} d s\right)$.

Proof:

1) This follows using the same techniques as in the proof of Theorem 4.1.

2) This follows by taking $t_{1}=\tau$ in Theorem 4.3, part 4). $\square$

While the conclusions of Corollary 4.4 give us exact generalizations of the results we obtained in the 3-node case, Corollary 4.4 is unsatisfactory because the additonal conditions of periodicity and persistency of excitation are, in general, uncheckable, except by simulations. Note that [28] has obtained similar results to Corollary 4.4 for a slightly more general RNN structure including self-connections of the neurons. More recently, one of the authors, see [29], has shown that if $z(\cdot)$ is periodic, then convergence of the weight error given in (24), and of $e_{n}(t)$ to zero, is exponential.

We illustrate the algorithm in the 5-node with $w^{*}=$ $(-1.0012,-1.0609,0.9491,1.4653)$. In the simulations we choose $z(0)=(-0.4170,-0.4739,-0.5049,-0.4906$, $-0.4056)$ for the teaching network, and $x(0)=(0,0,0$, $0,0)$ and $w(0)=(0,0,0,0)$ in the learning RNN and weight adaptation algorithm. The weights of the teaching network were chosen so that the linearization of (2) about $z=0$ has one pair of unstable complex conjugate eigenvalues and three exponentially stable eigenvalues. While we have been unable to prove that this type of eigenvalue configuration produces oscillatory behavior, our simulations suggest that this is the case. 

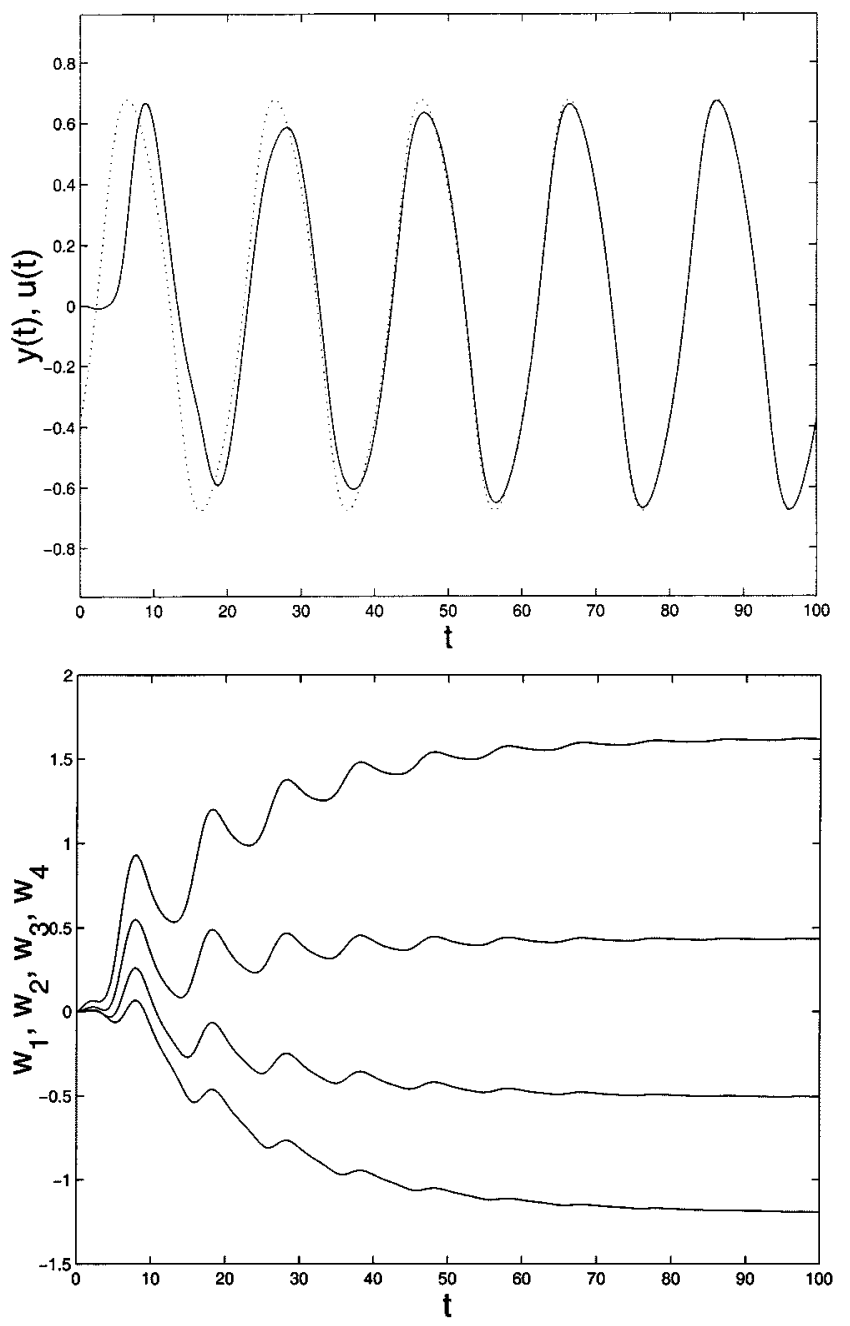

Fig. 3. (Top) reference signal $u(t)$ (dotted), learning signal $y(t)$ (continuous); (Bottom): weight dynamics $w_{1}(t), \cdots, w_{4}(t)$..

Fig. 3 shows the output signals and the weight convergence. In this simulation both the state error $x-z$ and the weight error $\tilde{w}$ converge to zero exponentially. This claim is supported by the fact that the matrix $\left(\int_{0}^{100} B(s) B(s)^{T} d s\right)$ is positive definite which, combined with the apparent periodicity of $z(\cdot)$, would give us the required persistency of excitation condition needed to apply Corollary 4.4 .

Remark 4.5: We discuss briefly the behavior of the replicating RNN. Under the persistency of excitation condition both the state and parameter error converge exponentially. So we can specify how small the errors are at the time, $T$, of switching from "learning" to "replication." Especially we can control the error between $w^{*}$ and $w(T)$. It is possible to show that the existence of attractive periodic orbits in the teaching network is an open property with regards to the weight values, and that the attractive periodic orbits depend continuously (with respect to the Hausdorff metric) on the weights, see [29]. This attractivity and continuous dependence are essentially nonlinear phenomena and suggests that our nonlinear RNN's have advantages over linear networks, such as those considered in [4] and [18]. In the nonlinear case, even though the outputs of the teaching network and replicating RNN may drift in phase, the corresponding
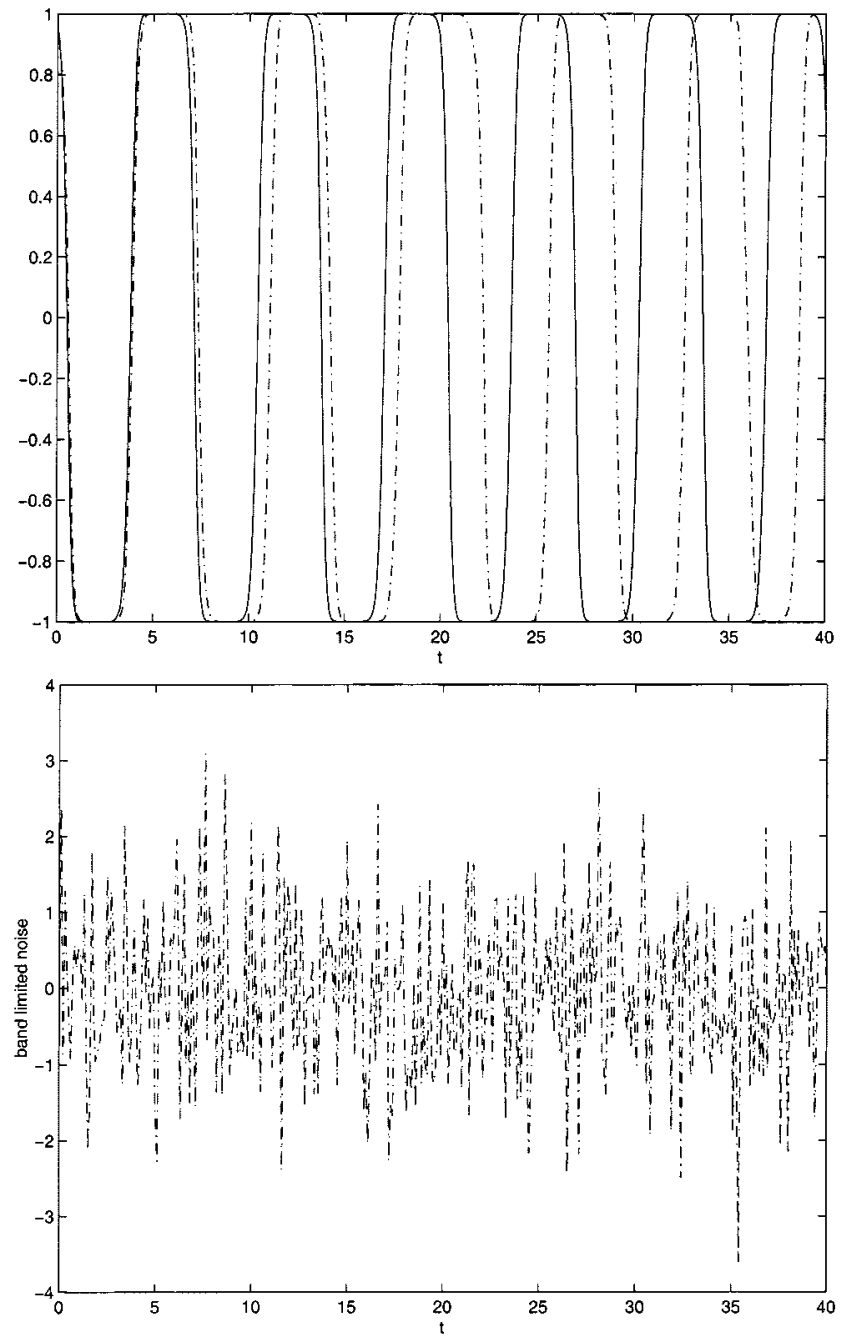

Fig. 4. (Top) Output of replicating RNN (1) without noise (solid) and with additive band-limited noise (dash-dotted). (Bottom) band-limited noise.

orbits remain close in state space. In the case of linear RNN's the identity, rather than tanh, is used as the triggering function and the resulting replicating RNN can be written as a linear system

$$
\dot{x}=A(w) x
$$

where the system matrix, describing the same connection structure as in Fig. 1, depends linearly on the weights. In this linear case, periodic solutions of the teaching network correspond to eigenvalues of $A\left(w^{*}\right)$ on the imaginary axis. However, given any $\delta>0$ there exists $w$ with $\left\|w-w^{*}\right\|<\delta$ and such that the eigenvalues of the replicating RNN have positive real parts. Consequently, if $w(T)=w$ then orbits of the teaching network and replicating RNN will diverge exponentially.

In Fig. 4 we illustrate this robustness with a simulation of a 3-node replicating RNN subject to band-limited noise $n(t)$ in the feedback loop. The weights of (1) in replicating mode are fixed so that $w_{1}=-20, w_{2}=10$. The noise is introduced additively so that $u(t)=\tanh x_{3}(t)+n(t)$. We see that the primary effect of the noise is to shift the phase of the signal. The shape of the signal is essentially retained. 


\section{Further COMments ON WeIGHT CONVERGENCE}

In Section III we proved that the 3-node teaching network has periodic solutions. In Section IV we proved that the learning RNN can learn these periodic outputs in the sense that the output error and weight error converge to zero exponentially. The proof of this exponential convergence used persistency of excitation conditions. For $n$-node RNN's, we could only obtain partial convergence results. There are two major obstacles to obtaining a general theory in the $n$-node case. First, we have not been able to determine conditions on the weights of the $n$-node teaching network (2) which guarantee the existence of periodic solutions. The existence of periodic solutions is fundamental to our idea of learning by repetition. Second, we have not been able to prove that $\left(\tanh z_{1}(\cdot), \cdots, \tanh z_{n-1}(\cdot)\right)^{T}$ is persistently exciting, or, equivalently in the case when $z(\cdot)$ is periodic, that the functions $\tanh z_{1}(\cdot), \cdots, \tanh z_{n-1}(\cdot)$ are linearly independent.

Our simulations suggest that linear independency, and hence the persistency of excitation condition, holds generically amongst those weight parameters for which the teaching network (2) has periodic solutions. However, there do exist teaching networks which have periodic solutions but which violate the linear independency condition. To construct such an example we need to modify (2) slightly by replacing the triggering function $a \mapsto \tanh a$ with $a \mapsto \tanh \lambda a$ for some $\lambda>0$.

Proposition 5.1: Let $z(t)$ be given by (2) but with triggering function $a \mapsto \tanh \lambda a$ for some $\lambda>0$. Assume $w_{k}^{*}=-1$ for some $k \in\{1, \cdots, n-1\}$ and $w_{j}^{*}=0$ otherwise.

1) If $\lambda>1$, then the modified teaching network possesses a limit cycle.

2) If $z(\cdot)$ is a periodic solution of the modified teaching network, then $z_{n-j} \equiv-z_{k-1-j}$ for $j=0, \cdots, k-2$. In particular, for $n \geq 4$, if $k \geq 3$, then the functions $\sigma\left(z_{1}(\cdot)\right), \cdots, \sigma\left(z_{n-1}(\cdot)\right)$ are linearly dependent.

Proof:

1) Let $F(z)=\left(f_{1}(z), \cdots, f_{n}(z)\right)^{T}$ denote the right-hand side of the modified teaching network. Consider first the case $k=1$. Then the teaching network forms a cyclic system (where components are indexed modulo $n$ ) with

$$
\prod_{j=1}^{n}\left|\frac{\partial f_{j}}{\partial z_{j+1}}(0)\right|=\lambda^{n}>1 .
$$

The existence of a limit cycle follows from [2] using techniques from [8].

Let $k>1$. Then the dynamics for components $z_{k}, \cdots, z_{n}$ are given by a cyclic $n-k+1$-dimensional subsystem which, as in the case $k=1$, has a limit cycle. For the other $k-1$ components let

$$
X=\{f: \mathbb{R} \rightarrow \mathbb{R}, f \text { continuous and periodic }\}
$$

and define a nonlinear operator $P: X \rightarrow X$ by

$$
(P f)(t)=\int_{-\infty}^{t} \exp (-(t-\tau) \sigma(f(\tau)) d \tau
$$

with $f \mapsto P^{j}(f)$ denoting $P$ composed with itself $j$ times. If $z_{k}, \cdots, z_{n}$ are the component functions of the limit cycle for the $n-k+1$-dimensional subsystem extended to $\mathbb{R}$ by periodicity, then the periodic functions $z_{k-1}=P\left(z_{k}\right), \cdots, z_{1}=P^{k-1}\left(z_{k}\right)$ restricted to $[0, \infty)$, determine the remaining $k-1$ components of the solution and hence the required limit cycle for the modified teaching network.

2) By the structure of the modified teaching network

$$
z_{j-1}(\cdot)=\left(P z_{j}\right)(\cdot) \quad \text { for } j=2, \cdots, n
$$

and

$$
z_{j}(\cdot)=\left(P^{n-j} z_{n}\right)(\cdot) \quad \text { for } j=1, \cdots, n \text {. }
$$

On the other hand using $P(-f)=-P f$ in the $n$th equation of the modified teaching network gives,

$$
\begin{aligned}
z_{n}(\cdot) & =\left(P\left(-z_{k}\right)\right)(\cdot)=-\left(P z_{k}\right)(\cdot) \\
& =-\left(P^{n-k+1} z_{n}\right)(\cdot)=-z_{k-1}(\cdot) .
\end{aligned}
$$

Applying $P^{j}$ to (32) and using (30)-(32), we obtain

$$
\begin{aligned}
z_{n-j}(\cdot) & =\left(P^{j} z_{n}\right)(\cdot)=\left(P^{j}\left(-z_{k-1}\right)\right)(\cdot) \\
& =-z_{(k-1)-j}(\cdot) \quad \text { for } j=0, \cdots, k-2 .
\end{aligned}
$$

This gives a very simple linear dependence of the $z_{j}$. Oddity of tanh $(\cdot)$ yields

$\sigma\left(z_{n-j}(\cdot)\right)=-\sigma\left(z_{k-1-j}(\cdot)\right) \quad$ for $j=0, \cdots, k-2$.

For a modified teaching network of dimension $n \geq 4$ with $k \geq 3$ the resulting dependency of the functions $\sigma\left(z_{1}(\cdot)\right), \cdots, \sigma\left(z_{n-1}(\cdot)\right)$ means that the corresponding persistency of excitation condition fails. This in turn means that exponential convergence of the weights cannot be guaranteed. It is quite delicate to actually find suitable parameters by which failure of exponential convergence of weights is observed. The failure of exponential convergence does occur in the case $n=5$ and $k=3$ with $\lambda=3$, for which, by Proposition 5.1, $z_{2}(\cdot)=-z_{5}(\cdot)$ and $z_{1}(\cdot)=-z_{4}(\cdot)$. For the simulation we choose $z(0)=(0.3975,0.4463,-0.0064,-0.3975,-0.4463)^{T}$, $x(0)=(0.3,0.1,0.2,-0.1,0.3)^{T}$ and $w(0)=(-0.05,0.1$, $-0.3,0.1)^{T}$. In this simulation the components $\tilde{w}_{2}$ and $\tilde{w}_{3}$ converge to zero exponentially and, on the time scale of the simulation, are almost indistinguishable. The components $\tilde{w}_{1}$ and $\tilde{w}_{4}$ do not converge to zero. Notice the strange behavior of $\left|\tilde{w}_{4}(t)\right|$ which appears to converge, to zero but then, after $t=500$, rises to a nonzero value.

Remark 5.2:

1) For $w_{k}^{*}=+1$ in Proposition 5.1, the modified teaching network is a cooperative system and therefore it cannot have a limit cycle. See [9].

2) While the modified cyclic teaching network is useful in illustrating that failure of the persistency of ex- 


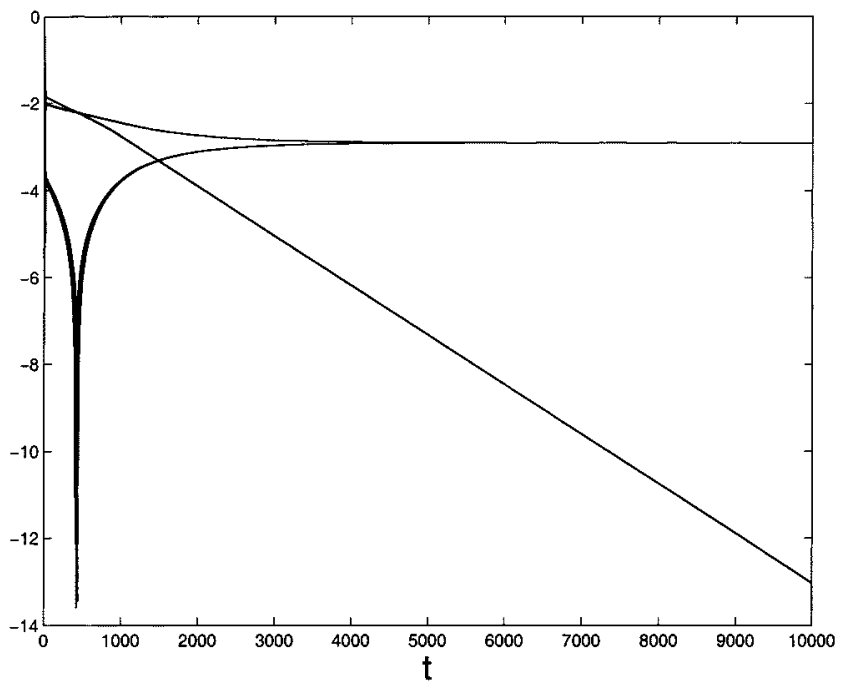

Fig. 5. Logarithm of weight error for a five-dimensional cyclic system.

citation can lead to nonconvergence of the weights, from the point of view learning its significance is limited. This is due to the fact that the components $z_{1}(\cdot), \cdots, z_{k-1}(\cdot)$ do not contribute to the dynamics of the output neuron $z_{n}(\cdot)$ in the teaching network. Hence the same output can be generated by a cyclic teaching network of dimension $n-k+1$ which, in all our simulations, yields a linearly independent set of functions $\left\{\tanh z_{k}(\cdot), \cdots, \tanh z_{n-1}(\cdot)\right\}$.

\section{CONCLUSIONS}

We have shown, using a result from monotone dynamical systems theory, that a certain 3-node RNN with fixed weights, the so-called teaching network, has periodic solutions. The motivation behind the need for the teaching network to have periodic solutions arises from the observation that learning usually requires repetition. We then used the periodic output of the teaching network as a teaching signal to be learned by a 3-node learning RNN. The learning RNN has a similar structure to the teaching network but with time-varying weights. The algorithm by which the weights are adapted is similar to parameter identification algorithms in adaptive control. We were able to prove global exponential convergence of the state and weights of the learning RNN to the fixed weights and periodic solution of the teaching network. This global and exponential convergence is much sharper than the local and asymptotic convergence which is usually associated with gradient descent adaptation. Note also that the inherently nonlinear nature and the resulting limit cycle-like structure of the periodic solutions of the teaching network and learning/replicating RNN provides robustness of the learned signal against external disturbances. This contrasts with the case of linear RNN's as developed by [4] and [18] which are sensitive to such disturbances. We also obtained partial convergence results in the $n$-node case by using techniques from adaptive control. Under appropriate persistency of excitation type conditions we obtain global exponential convergence as in the 3-node case.
Techniques for speeding up the exponential convergence of the weights in the case when the persistency of excitation condition is satisfied have been developed in [28] for a similar RNN structure. These techniques could also be applied to our class of RNN.

Applications of our results to the control of a robot arm have been developed in [21], in the case of a gradient descent weight adaptation algorithm, and in [26], with our weight adaptation algorithm.

An area of research which requires further work is to make use of monotone dynamical systems theory in studying more general RNN structures. So far our results are restricted, in the main, to a special class 3-node RNN's. Another issue, which we did not address here, is to understand the detailed structure of the class of periodic signals which can be generated by the teaching network. Our simulations suggest that the periodic signals are very nearly sinusoidal. This issue would be important if many learning RNN's were combined in parallel so as to facilitate learning of more complicated signals. See [20] for preliminary simulation-based studies of RNN's comprised of several 3-node networks in parallel.

Finally, we have restricted attention to the problem of learning and then replicating a teaching signal. Another issue of interest is to consider the recall capabilities of RNN's. More precisely, how can we build into the learning RNN, mechanisms for recognizing a previously learned signal so as to then speed-up, or even bypass, relearning. For results in this direction see [27].

\section{REFERENCES}

[1] H. Asada and J. J. E. Slotine, Robot Analysis and Control. New York: Wiley, 1986.

[2] A. Atiya and P. Baldi, "Oscillations and synchronizations in neural networks: An exploration of the labeling hypothesis," Int. J. Neural Syst., vol. 1, pp. 103-124, 1989.

[3] A. Babloyantz, C. Lourenco, and A. J. Sepulchre, "Control of chaos in delay differential equations in a network of oscillators and in model cortex," Phys. D, vol. 86, pp. 274-283, 1995.

[4] R. Blach and D. H. Owens, "Replication of periodic signals using a neural-type structured network," Centre for Systems and Control Engineering, University of Exeter, Int. Rep. 92/03, 1992.

[5] S. Boyd and S. S. Sastry, "Necessary and sufficient conditions for parameter convergence in adaptive control," Automatica, vol. 22, pp. 629-639, 1986.

[6] K. Doya and S. Yoshizawa, "Adaptive neural oscillator using continuous time backpropagation learning," Neural Networks, vol. 2, pp. 375-385, 1989.

[7] B. Doyon, M. Cessac, M. Quoy, and M. Samuelides, "Control of the transition to chaos in neural networks with random connectivity," Int. J. Bifurcation Chaos, vol. 3, pp. 279-291, 1993.

[8] S. Hastings, J. J. Tyson, and D. Webster, "Existence of periodic solutions for negative feedback equations," J. Differential Equations, vol. 25, pp. 39-64, 1977.

[9] M. Hirsch, "Systems of differential equations which are competitive or cooperative III," Nonlinearity, vol. 1, pp. 51-71, 1988.

[10] R. A. Horn and C. R. Johnson, Matrix Analysis. Cambridge: Cambridge Univ. Press, 1985.

[11] Z. Li and J. J. Hopfield, "Modeling the olfactory bulb and its neural oscillatory processings,” Biol. Cybern., vol. 61, pp. 379-392, 1989.

[12] J. Mallet-Paret and H. Smith, "The Poincaré-Bendixson theorm for monotone cyclic feedback systems," J. Differential Equations, vol. 2, pp. 376-421, 1990.

[13] J. Mallet-Paret and G. R. Sell, "The Poincaré-Bendixson theorem for monotone cyclic feedback systems with delay," J. Differential Equations, vol. 125, pp. 441-489, 1996. 
[14] A. P. Morgan and K. S. Narendra, "On the stability of nonautonomous differential equations $\dot{x}=[A+B(t)] x$, with skew symmetric matric $B(t)$," SIAM J. Contr. Optimization, vol. 15, pp. 163-176, 1977.

[15] K. S. Narendra and A. Annaswamy, Stable Adaptive Systems. Englewood Cliffs, NJ: Prentice-Hall, 1987.

[16] K. S. Narendra and K. Parthasarathy, "Identification and control of dynamical systems using neural networks," IEEE Trans. Neural Networks, vol. 1, pp. 4-26, 1990.

[17] B. A. Pearlmutter, "Gradient calculations for dynamic recurrent neural networks: A survey," IEEE Trans. Neural Networks, vol. 6, pp. $1212-1228,1995$.

[18] R. Reinke, "Adaptive regeln zum lernen und reproduzieren periodischer signale mit dynamischen netzwerken," Ph.D. dissertation, Universität Kaiserslautern.

[19] W. J. Rugh, Linear Systems Theory. Englewood Cliffs, NJ: PrenticeHall, 1996.

[20] A. Ruiz, D. H. Owens, and S. Townley, " $L_{2}$ approximation properties of recurrent neural networks," in Proc. 4th European Contr. Conf. (Electron. Publication WE-E G1), Brussels, July 1997.

[21] — "Existence, learning and replication of periodic motions in recurrent neural networks," IEEE Trans. Neural Networks, vol. 9, pp. $651-661,1998$.
[22] S. Sastry and M. Bodson, Adaptive Control: Stability, Convergence and Robustness. Englewood Cliffs, NJ: Prentice-Hall, 1979.

[23] H. Smith, "Convergene and oscillatory activation dynamics for cascades of neural nets with nearest neighbor competitive or cooperative interactions," Neural Networks, vol. 4, pp. 41-46, 1991.

[24] — Monotone Dynamical Systems. Providence, RI: Amer. Math. Soc., 1995.

[25] R. V. Sole and L. M. de la Pride, "Controlling chaos in discrete neural networks," Phys. Lett. A, vol. 199, pp. 65-69, 1995.

[26] R. Waschler, A learning controller for lineariable nonlinear systems.

[27] R. V. Sole and L. M. de la Prida, "Controlling chaos in discrete neural networks," Phys. Lett. A, vol. 199, pp. 65-69, 1995.

[28] M. G. Weiss, "Learning oscillations using adaptive control," Proc. ICANN 97, vol. LNCS 1327, pp. 331-336, 1997.

[29] _ _ "Learning periodic signals with recurrent neural networks," $\mathrm{Ph} . \mathrm{D}$. dissertation, Fachbereich Math., Universität Kaiserslautern, 1999.

[30] H. Yang and T. S. Dillon, IEEE Trans. Neural Networks, vol. 5, pp. 719-729, 1994 